\title{
Systematic Study and Meta-Analysis of Risk Factors Associated with Cholera Outbreaks in Africa: A Review
}

\author{
Nupolu Giftee Paye ${ }^{1 *}$, Robert H. B. Miller ${ }^{2}$, Chengchao Zhou ${ }^{1}$, Sonkarlay J. Y. Weamie ${ }^{3}$, \\ Tito A. Davies 4 \\ ${ }^{1}$ School of Public Health, Cheeloo College of Medicine, Shandong University, Jinan, China \\ ${ }^{2}$ College of Safety and Environmental Engineering, Shandong University of Science and Technology, Qingdao, China \\ ${ }^{3}$ Hunan University, Changsha, China \\ ${ }^{4}$ Stella Maris Polytechnic University, Monrovia, Liberia \\ Email: ^nupolugpaye@gmail.com, roberlynehbmiller@gmail.com, ^zhouchengchao@sdu.edu.cn, skweamie@hnu.edu.cn, \\ tadavies2002@gmail.com
}

How to cite this paper: Paye, N.G., Miller, R.H.B., Zhou, C.C., Weamie, S.J.Y. and Davies, T.A. (2021) Systematic Study and Meta-Analysis of Risk Factors Associated with Cholera Outbreaks in Africa: A Review. $A d$ vances in Infectious Diseases, 11, 240-260. https://doi.org/10.4236/aid.2021.112022

Received: May 19, 2021

Accepted: June 26, 2021

Published: June 29, 2021

Copyright $\odot 2021$ by author(s) and Scientific Research Publishing Inc. This work is licensed under the Creative Commons Attribution International License (CC BY 4.0).

http://creativecommons.org/licenses/by/4.0/

(c) (i) Open Access

\begin{abstract}
In Sub-Saharan Africa, cholera is a persistent public health problem. Various methods are now being applied to reduce the mobility and mortality that these diseases cause. Because of the implemented policies, there seem to have been several improvements. However, the constraints suggest inefficiency in eliminating the burden, necessitating a straightforward explanation. The systematic review and meta-analysis aimed are to investigate the reasons for the incomplete eradication of cholera disease in Sub-Saharan Africa. We were able to obtain pooled estimates using random effect models. Four experiments were excluded from the study because they were duplicated in the studies, 13 because of an outbreak did not occur, 13 publications did not include risk factors and odds ratios in their outcome analysis, and an outbreak occurred before 2013. The review points out that drinking contaminated water, consuming unwashed vegetables, and the use of poor publish toilet facilities enhance cholera in Africa. PubMed, Web of Science, EMBASE, and EBSCO were among the four meticulously and systematically searched databases using predetermined criteria or search strategies. Two independent reviewers recognized studies that met the selection criteria, and data was collected using validated methods.
\end{abstract}

\section{Keywords}

Cholera, Sub-Saharan Africa, Risk Factors, Improved Water, Hygiene Services 


\section{Introduction}

This public health burden continues to exist in deprived areas worldwide as an ancient and seemingly neglected disease. In 1817, there was the first Cholera epidemic [1]. Six more pandemics have occurred since then, and outbreaks have yet to be contained. Inadequate health literacy, inadequate water and sanitation services, community mobilization, the absence of national plans, cross-border partnerships, and overcrowding are all factors that increase the risk of cholera [2] [3]. Studies have shown that herd immunity does not exist when it comes to the battle against cholera in Sub-Saharan Africa [4] [5]. For example, non-endemic areas of the Democratic Republic of Congo have higher case-fatality rates than endemic areas (DRC) [6]. It is due to a lack of immunity and less effective prevention and care [7].

Over the last two decades, there has been a steady improvement in infant survival rates. This performance, however, can only be calculated at the national level; localized improvement is still unknown [8]. The global prevalence of diarrheal diseases is estimated to be between 1.7 and 5 billion cases per year [9]. Cholera outbreaks are more likely in low- and middle-income countries than in developed countries where housing is overcrowded, and water and sanitation are inadequate, thereby, increasing death rate, meta illness and poor health leading to gastrointestinal disfunction [9] [10].

Using Liberia as an example, children under 18 are subjected to hardship that negatively impacts their lives, minds, wellbeing, and emotions.

Multi-approach interventions are often needed. For example, according to UNICEF Liberia 2018, one-third of the country's children are under five [11].

Most of these children are also not receiving any of the necessary assistance.

With a mortality rate of 1072 deaths per 100,000 live births, many children are born without a mother to look after them. During the initial six months of the lactating period, about one out of every two babies is exclusively breastfed.

Although teenage mothers are blamed for the high rates of maternal and neonatal deaths, killer diseases such as malaria, pneumonia, measles, AIDS, and diarrhea kill one out of every ten children before they reach five. In addition, typhoid fever (TF) and cholera are two of the most highly life-threatening infectious diseases [12].

Cholera increases the risk of death in children and older people than adults [13]. The Global Enteric Multicenter Analysis conducted this research (GEMS).

The GEM research [14] was a three-year case-control study that looked at the burden, etiology, and effects of moderate-to-severe diarrhea (MSD). Cholera is prevalent in several African countries, especially the Horn of Africa and the Arabian Peninsula.

Corresponding to the United Nations Sustainable Development Goal 3.2, multi-pronged measures are expected to eliminate preventable child deaths by 2030 [15] [16]. 
The data tabulated from the national study is insufficient. The best deciding factors are steady estimates of infant mortality rates and patterns at the subnational level [8]. Within 99 low- and middle-income countries, the sub-national difference in mortality rates and the number of deaths of neonates and children below five years is not examined [8]. In 2018, a cholera outbreak in Nigeria's northern region signaled an increased burden. It emphasizes the strategic importance of a multi-sectoral strategy in developing and implementing public health strategies [17].

A geostatistical survival model estimated that 32 percent of children under five lived in districts with infant mortality rates of 25 or less per 1000 live births by 2017. Around 2000 and 2017, 58 percent of child deaths were reported to have occurred [18] [19]. Cholera outbreaks across borders are a significant public health concern in Sub-Saharan Africa. It leads to the high number of cholera cases and deaths recorded every year. Both people's lives and livelihoods are impacted, making prevention and regulation more complex [20]. The most vulnerable age ranges are children under the age of five who are in kindergarten. As a result, guidelines and procedures should be checked to delegate specific roles and obligations to children under eighteen. It will ensure that these epidemics are effectively prevented, detected, monitored, and controlled [20] [21].

Prevention by better water, sanitation, hygiene facilities, and the use of oral cholera vaccines (OCVs) [22] [23] are only a few of the methods that have been used.

In Sub-Saharan Africa, however, there are outbreaks and occasional cases of cholera.

We were unable to locate a report that attempted to quantify the global prevalence of the burden to the best of our knowledge. Subsequently, we conducted a systematic review and meta-study to investigate why the incomplete eradication of cholera diseases in Sub-Saharan Africa.

\section{Materials and Method}

The current research was conducted between December 2020 and February 2021 as a systematic overview and meta-analysis. This study supported the Preferred Reporting Items for Systematic Reviews and Meta-Analysis guidelines (PRISMA). The goal was to find a pooled estimate of the odds ratio of risk factors linked to African cholera outbreaks. In addition, the research also looked at the most common risk factor associated with cholera outbreaks in the general population in Africa.

\subsection{The Search Strategy}

A thorough and systematic search of four databases (PubMed, EMBASE, Web of Science, and EBSCO) was conducted. The search was restricted to 1990 through 2020 , with the most recent search taking place on January 25, 2021. Using the 
following search technique, we conducted advanced searches in all databases using MeSH (Medical Subject Headings) on PubMed ((“"Risk Factors" [Mesh]) and "Cholera" $[$ Mesh]) "Africa South of the Sahara" [Mesh]). Moreover, TS = Risk factors AND TS = cholera AND TS = Africa are used for Web of Science, ("risk factor"/exp OR "risk factor") AND ("cholera"/exp OR cholera) AND ("Africa south of the site For studies conducted in English, the researcher also included keywords such as "cholera," "outbreaks," "risk factors," "Vibrio cholera," and "Africa."

\subsection{Requirement for Eligibility and Exclusion}

The following criteria were used to select inclusion criteria: observational epidemiological studies, including case management, cohort studies, cross-sectional (retrospective or prospective studies) with a population-based design that quantifies or defines risk factors associated with cholera outbreaks in any age group [24]. A risk factor is characterized as an aspect of personal behavior or lifestyle, environmental exposure, an inborn or hereditary trait known to be correlated with a health-related condition that is important to avoid based on epidemiological evidence (MeSH definition). Participants in the study must be from Afri$\mathrm{ca}$, and the research must be performed or published in English. The risk factors that are included in the study must be listed as odds ratios. Studies were excluded if the study's result did not match the study's goal, and studies that used mixed methods were also excluded. The risk factor identified in the studies was used to test them.

\subsection{Extraction of Information}

Two investigators (S.F.M. and UB) carried out retrieving studies, who worked independently on the searches and handled the findings using EndNote X9 (Build 12 062). After eliminating duplicates, the reviewers looked at the titles of the retrieved papers to see if they were necessary. In addition, for full-text articles, specific articles were sought and screened for inclusion based on the inclusion criteria. When inconsistencies arose during the selection process, the third author resolved them. Author's name, year of publication, country, age range, study design, sample size, cases, controls, odds ratio, and confidence intervals of risk factors were all extracted from each paper using a standardized form with the following domains: author's name, year of publication, country, age range, study design, sample size, cases, controls, odds ratio and confidence intervals of risk factors.

\subsection{Statistical Analysis}

The risk factors associated with cholera outbreaks were the only outcome of this research. The established risk factors are examined individually, with subgroup analysis by region. The impact sizes and standard errors were calculated using StataCorp.16.0 commands for meta-analysis, using the risk factors' odds ratios and confidence gaps. The odds ratios for the individual hazard factors were cal- 
culated using a pooled calculation. The standard error, confidence interval, and forest plots were calculated using STATA version 16 software. Depending on the heterogeneity, the random-effects model was used. The Cochran Q-test of heterogeneity was used to assess statistical heterogeneity in the studies at a significance level of 5\%, and I2 was used to assess quantitative heterogeneity among the findings according to the Higgins classification, with an I2 value above $75 \%$ indicating significant heterogeneity.

Furthermore, the Begg and Egger experiments were used to look at publication bias. In the case of high heterogeneity, the cause of heterogeneity was investigated, and sensitivity analysis was carried out by eliminating one study from the analysis and studies of inadequate consistency.

\subsection{Evaluation of the Included Study Quality}

The methodological authenticity of the included studies was determined using the JBI. Critical Appraisal Checklist for Cohort Studies and the JBI, Critical Appraisal Checklist for Case-Control Studies. The studies' nature, execution, and interpretation were scrutinized for the possibility of bias. The checklist had ten questions with yes, no, ambiguous, and not applicable answers. The frequency of yes and no responses determines high and low quality, respectively. Uncertain answers necessitate contacting the relevant author for clarification. The papers that were included were evaluated using the following questions: Are the groups comparable except for the presence of disease in cases of the absence of disease in controls, were cases and controls appropriately matched, were the same criteria used for case and control identification, was exposure measured in a standard, valid, and reliable manner, was exposure measured in the same manner for cases and controls, where confounding factors identified, and were strategies implemented? Participant recruitment, study design, and sample size were carefully examined for potential bias in the papers. The ten included studies all used a case-control study design. However, one of the ten trials did not have enough participants (Table 1).

\section{Result}

Our quest yielded 460 papers found using electronic search engines and strategies. Out of these, 97 were eliminated as duplicates, leaving 363 articles for further screening by title, full text, and abstract, which resulted in the exclusion of 321 more articles. As a result, 42 full-text studies were screened for inclusion criteria and other parameters aligned with the review objectives. Four findings were omitted from the research because they were not English studies, 14 were outside of the study environment, and thirteen papers did not include risk factors and odds ratios in Figure 1.

\subsection{Result Characteristic}

Eleven papers were included in the final systematic review and meta-analysis, 
Table 1. Results of the Quality assessment of included studies using JBI checklist for case-control studies.

\begin{tabular}{|c|c|c|c|c|c|c|c|c|c|c|}
\hline & 1 & 2 & 3 & 4 & 5 & 6 & 7 & 8 & 9 & 10 \\
\hline Nsagha et al. 2015 [25] & $\checkmark$ & $\checkmark$ & $\checkmark$ & $\checkmark$ & $\checkmark$ & $\checkmark$ & $\checkmark$ & $\checkmark$ & $\checkmark$ & $\checkmark$ \\
\hline Dined et al. 2020 [26] & $\checkmark$ & $\checkmark$ & $\checkmark$ & $\checkmark$ & $\checkmark$ & + & UC. & $\checkmark$ & $\checkmark$ & $\checkmark$ \\
\hline Hudson et al. 2020 [27] & $\checkmark$ & $\checkmark$ & $\checkmark$ & $\checkmark$ & $\checkmark$ & + & UC. & $\checkmark$ & $\checkmark$ & $\checkmark$ \\
\hline Saheed et al. 2018 [28] & $\checkmark$ & $\checkmark$ & $\checkmark$ & $\checkmark$ & $\checkmark$ & $\checkmark$ & $\checkmark$ & $\checkmark$ & $\checkmark$ & $\checkmark$ \\
\hline Nwafor et al. 2019 [27] & $\checkmark$ & $\checkmark$ & $\checkmark$ & $\checkmark$ & $\checkmark$ & $\checkmark$ & $\checkmark$ & $\checkmark$ & $\checkmark$ & $\checkmark$ \\
\hline Thomas T. et al. 2015 [29] & $\checkmark$ & $\checkmark$ & $\checkmark$ & $\checkmark$ & $\checkmark$ & + & $\checkmark$ & $\checkmark$ & $\checkmark$ & $\checkmark$ \\
\hline Francis et al. 2017 [30] & $\checkmark$ & $\checkmark$ & $\checkmark$ & $\checkmark$ & $\checkmark$ & + & UC. & $\checkmark$ & $\checkmark$ & $\checkmark$ \\
\hline Fatigue et al. 2013 [31] & $\checkmark$ & $\checkmark$ & $\checkmark$ & $\checkmark$ & $\checkmark$ & + & UC. & $\checkmark$ & $\checkmark$ & $\checkmark$ \\
\hline Kone et al. 2008 [32] & $\checkmark$ & $\checkmark$ & $\checkmark$ & $\checkmark$ & $\checkmark$ & $\checkmark$ & $\checkmark$ & $\checkmark$ & $\checkmark$ & $\checkmark$ \\
\hline Alvin Shultz et al. 2009 [33] & $\checkmark$ & $\checkmark$ & $\checkmark$ & $\checkmark$ & $\checkmark$ & + & UC. & $\checkmark$ & $\checkmark$ & $\checkmark$ \\
\hline Assen et al. 2019 [34] & $\checkmark$ & $\checkmark$ & $\checkmark$ & $\checkmark$ & $\checkmark$ & + & UC. & $\checkmark$ & $\checkmark$ & $\checkmark$ \\
\hline
\end{tabular}

Note: 1 = were groups comparable, 2 = were cases and controls matched, and $3=$ were the same criteria used to identify cases and controls. $4=$ were exposure measured in a standard way, $5=$ were exposure restrained in the same way for cases and controls, $6=$ were bewildering factors recognized, 7 were approaches to deal with confounding factors mentioned, $8=$ were outcomes assessed in a standard way in addition, $=$ Yes and $=$ No, UC $=$ undefined, and NA $=$ not available are used.

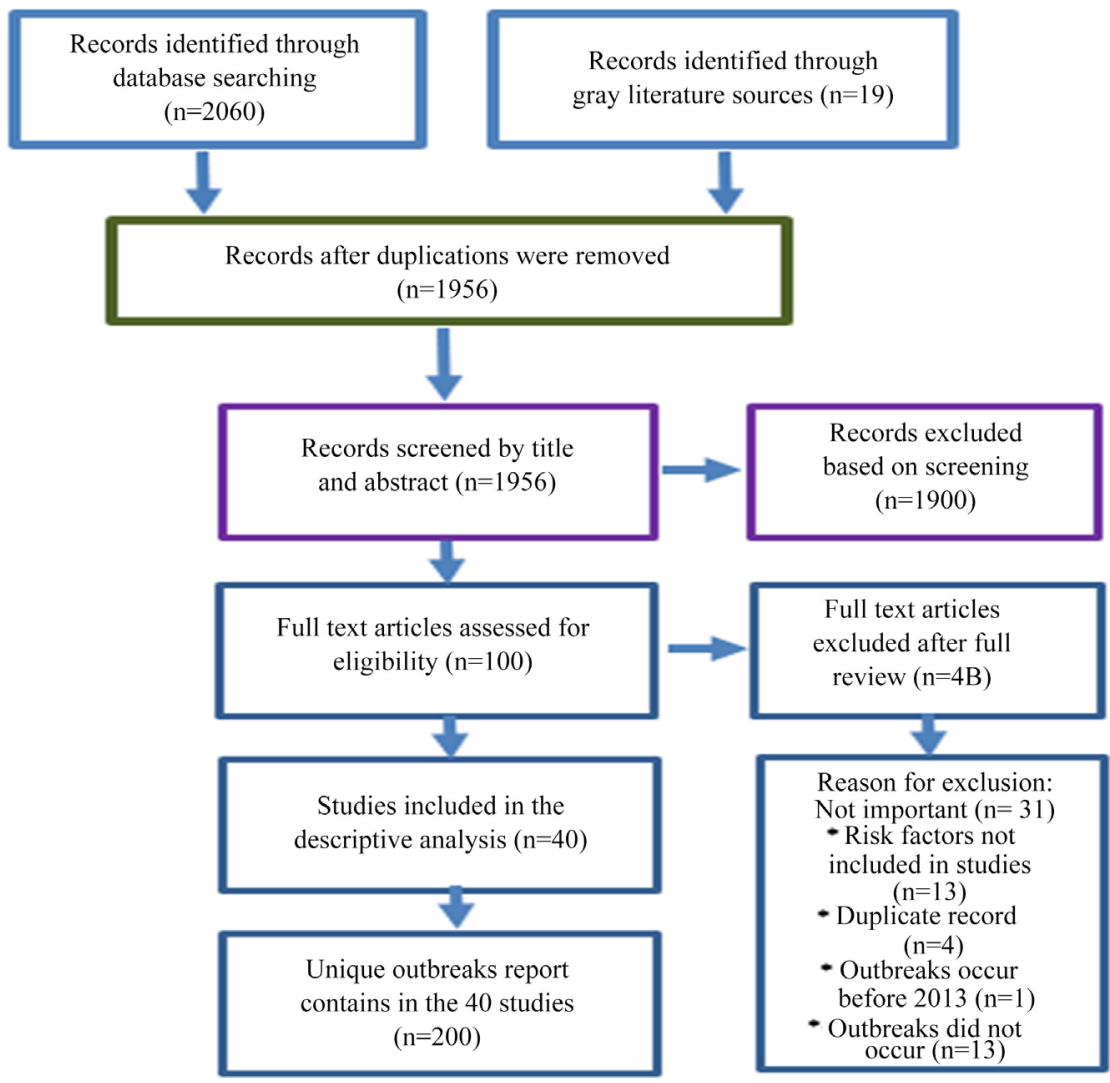

Figure 1. PRISMA gush diagram detailing the study selection process of included studies. 
which employed three different research designs. The majority of the studies (8) used an unmatched case-control design, while three used matched case-control, one retrospective matched case-control, and one case-control design. Nigeria accounted for three total studies, while Ethiopia (2 studies), Kenya (2 studies), Cameroon, South Sudan, Zambia, and Zimbabwe accounted for one. The sample size for the participants ranged from 47 to 300 , and all of the studies included case and control groups.

Furthermore, most studies, cases, and controls were not equivalent, and data collection was done in the neighborhood Table 2. Drinking untreated water ( 7 studies), consuming raw vegetables (4 studies), interaction with an infected person(s) (7 studies), eating outside of the home (4 studies), sharing toilet facilities with more than one household (4 studies), a lack of health education on preventive measures/intervention ( 3 studies), and poor hand hygiene before eating was established as risk factors (2 studies).

Table 2. Summary of the primary study data based on WHO 2013 Report, Afro region 2012 [35].

\begin{tabular}{|c|c|c|c|c|c|}
\hline \multirow{2}{*}{ Country } & \multicolumn{3}{|c|}{ Cholera Out Break } & \multirow{2}{*}{ Case Fatality ratio (\%) } & \multirow{2}{*}{ Preventive Measure } \\
\hline & Cases & Deaths & Meta Recovery & & \\
\hline DR Congo & 391,524 & 30,539 & 360,985 & 7.80 & Personal Hygiene \\
\hline Mozambique & 315,295 & 11,752 & 303,543 & 3.73 & Personal Hygiene \\
\hline Nigeria & 260,966 & 259,920 & 1046 & 9.96 & Personal Hygiene \\
\hline Tanzania & 204,569 & 172,938 & 31,631 & 8.45 & Personal Hygiene \\
\hline South Africa & 186,462 & 1426 & 185,046 & 0.76 & Personal Hygiene \\
\hline Angola & 182,875 & 13,326 & 169,551 & 7.29 & Personal Hygiene \\
\hline Zimbabwe & 153,335 & 13,783 & 139,552 & 8.99 & Personal Hygiene \\
\hline Malawi & 140,263 & 5428 & 134,845 & 3.87 & Personal Hygiene \\
\hline Ghana & 128,525 & 6400 & 122,125 & 4.98 & Personal Hygiene \\
\hline Ethiopia & 118,297 & 2846 & 116,451 & 2.41 & Personal Hygiene \\
\hline Uganda & 99,517 & 7630 & 91,887 & 7.67 & Personal Hygiene \\
\hline Kenya & 99,022 & 6556 & 92,466 & 6.62 & Personal Hygiene \\
\hline Zambia & 97,408 & 6698 & 90,710 & 6.88 & Personal Hygiene \\
\hline Guinea-Bissau & 91,609 & 3587 & 88,022 & 3.92 & Personal Hygiene \\
\hline Liberia & 84,999 & 964 & 84,935 & 1.13 & Personal Hygiene \\
\hline Cameroon & 72,551 & 5876 & 66,675 & 8.10 & Personal Hygiene \\
\hline Burundi & 31,990 & 1042 & 30,948 & 3.26 & Personal Hygiene \\
\hline Cape Verde & 14,144 & 285 & 13,859 & 2.01 & Personal Hygiene \\
\hline Algeria & 12,729 & 650 & 12,079 & 5.11 & Personal Hygiene \\
\hline Burkina Faso & 12,564 & 1513 & 1051 & 12.04 & Personal Hygiene \\
\hline Rwanda & 11,449 & 585 & 10,864 & 5.11 & Personal Hygiene \\
\hline Swaziland & 10,107 & 368 & 9739 & 3.64 & Personal Hygiene \\
\hline
\end{tabular}




\subsection{Meta-Analysis}

\subsubsection{Drinking Untreated Water}

Seven included studies identified the connection between drinking untreated water and cholera outbreaks. The research was carried out in Nigeria [36], Ethiopia [37], Cameroon [38], Kenya [39], and Zambia [40]. This risk factor for a cholera outbreak was correlated with a pooled odds ratio calculation shown in Figure 2 and Figure 3 [OR $=1.83$; 95 percent CI: $(1.23,2.44)]$. The heterogeneity $(\mathrm{I} 2=62.78 \%)$ between studies was less than $75 \%$.

\subsubsection{Eating Raw Vegetables}

Eating raw vegetables was the second risk factor associated with the cholera outbreak. This risk factor was the subject of four reports. The analysis has pooled odds ratio calculation [OR $=1.92$; 95 percent CI: $(0.88-2.97)$ ], the probability of cholera outbreak was nearly two times higher among people who consumed raw vegetables Figure 4.

\begin{tabular}{l} 
Study \\
\hline Nsagha et a. 2015 \\
Dinede et al. 2020 \\
Hudson et al 2020 \\
saheed et al 2018 \\
Dan-nwafor et al 2019 \\
Abdulha et al 2018 \\
Francis et al 2017 \\
Overall \\
Heterogeneity: $\mathrm{T}^{2}=0.39, \mathrm{I}^{2}=61.78 \%, \mathrm{H}^{2}=2.62$
\end{tabular}

Figure 2. The estimate of the pooled odds ratio differed by region. Cameroon $[\mathrm{OR}=2.219 ; 95$ percent CI: $(1.300-3.139)]$, Ethiopia [OR $=2.182$; 95 percent CI: $(1.525-2.839)]$, Kenya $[\mathrm{OR}=1.871$; 95 percent CI: $(.821$ 2.922)], Nigeria $[\mathrm{OR}=1.639 ; 95$ percent $\mathrm{CI}:(-0.406-3.685)]$ and Zambia $[\mathrm{OR}=0.875$; 95 percent $\mathrm{CI}:(0.062-1.689)]$ had the highest estimate.

\begin{tabular}{l} 
Study \\
\hline Nsagha et a. 2015 \\
Dinede et al. 2020 \\
Hudson et al 2020 \\
saheed et al 2018 \\
Dan-nwafor et al 2019 \\
Abdulha et al 2018 \\
Francis et al 2017 \\
Overall \\
Heterogeneity: $\mathrm{T}^{2}=0.39, \mathrm{I}^{2}=61.78 \%, \mathrm{H}^{2}=2.62$ \\
Test of $\theta=\theta_{\mathrm{j}}: \mathrm{Q}(6)=15.41, \mathrm{p}=0.02$
\end{tabular}

Figure 3. Forest plots of odds, ratio estimates of cholera outbreak by drinking untreated water. 


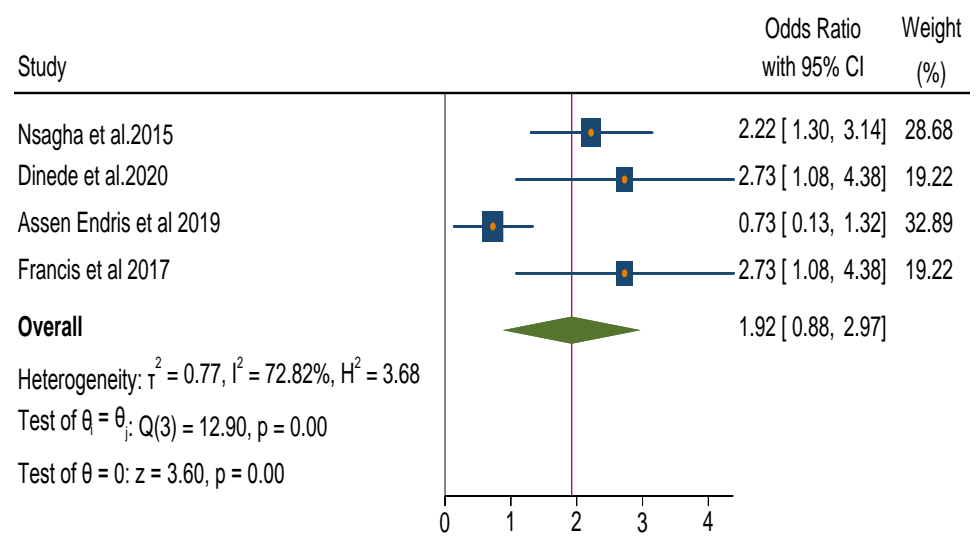

Figure 4. Forest plot of odds ratio estimates of cholera outbreak by eating raw vegetables.

\subsubsection{Contact with an Infected Individual}

Communication with an infected individual was classified as the third risk factor identified in the included articles. It was reported in seven papers as a risk factor associated with cholera outbreaks. The pooled odds ratio calculation $[\mathrm{OR}=1.78$; 95 percent CI: $(1.20-2.36)]$ revealed that individuals who come into contact with sick people are nearly twice as likely to catch the disease, shown in Figure 5 .

The degree of heterogeneity $(\mathrm{I} 2=42.76 \%)$ between studies was less than $75 \%$.

\subsubsection{Sharing Toilet Facilities with More Than One Person}

Sharing toilet facilities with more than one household was the fourth risk factor associated with cholera outbreaks. The pooled odds ratio calculation [OR $=1.09$; 95 percent CI: $(0.62-1.55)]$ was correlated with this risk factor for cholera outbreaks in four articles from four different countries Figure 6.

\subsubsection{Inadequate Health Education}

The fifth factor linked to cholera outbreaks among African populations was a lack of health education on preventive measures/interventions: three of the eleven studies published on this risk factor. The pooled odds ratio in Figure 7 calculation $[\mathrm{OR}=1.18 ; 95$ percent CI: $(0.72-1.64)]$ was correlated with cholera outbreak health education.

\subsubsection{Eating Outside of the Home}

Four researches looked at eating outside the home as a potential factor for cholera outbreaks. According to the pooled odds ratio calculation shown in Figure 8, [OR $=2.02$; 95 percent CI: $(1.28-3.18)]$, people who eat out are more expected to catch the disease than those at home. The heterogeneity between studies was 62.7 percent less than the threshold of significant heterogeneity of 75 percent.

\subsubsection{Eligibility of Hand Hygiene}

Just two of the included studies published on poor hand hygiene as a risk factor for cholera, and both were performed in Nigeria. This risk factor was correlated with the pooled odds ratio calculation $[\mathrm{OR}=0.62$; 95 percent CI: $(0.29-1.54)]$, 
which are shown in Figure 9. There was much variation between the studies (91 percent).

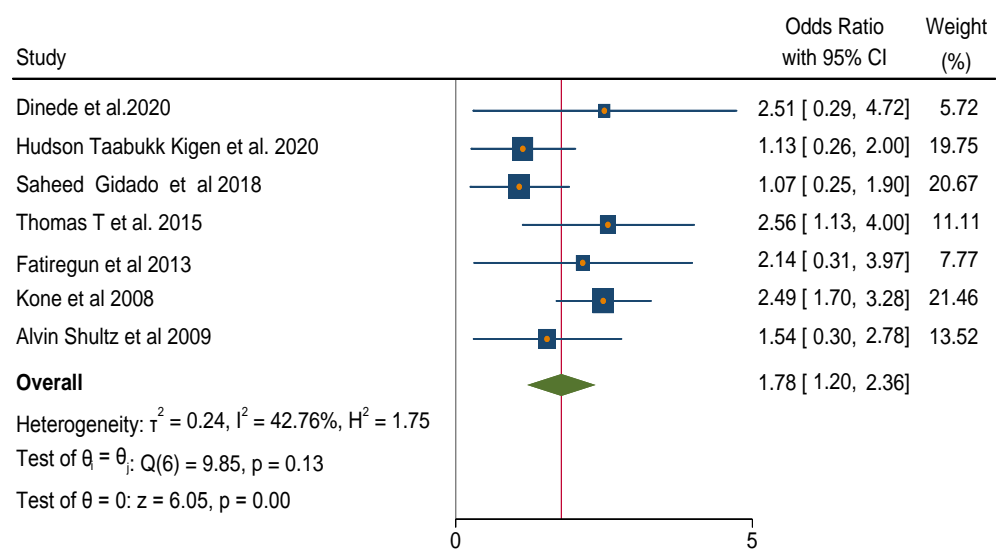

Random-effects REML model

Figure 5. Forest plot of odds, ratio estimates of a cholera outbreak due to contact with infected persons.

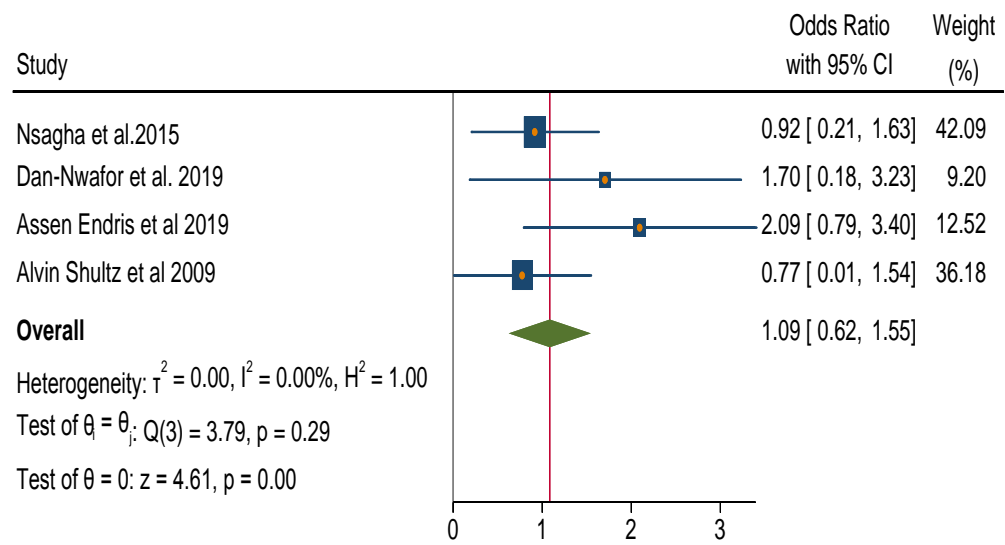

Random-effects REML model

Figure 6. A Forest plot of odds ratio estimates of a cholera outbreak due to sharing toilet facilities with more than one household.

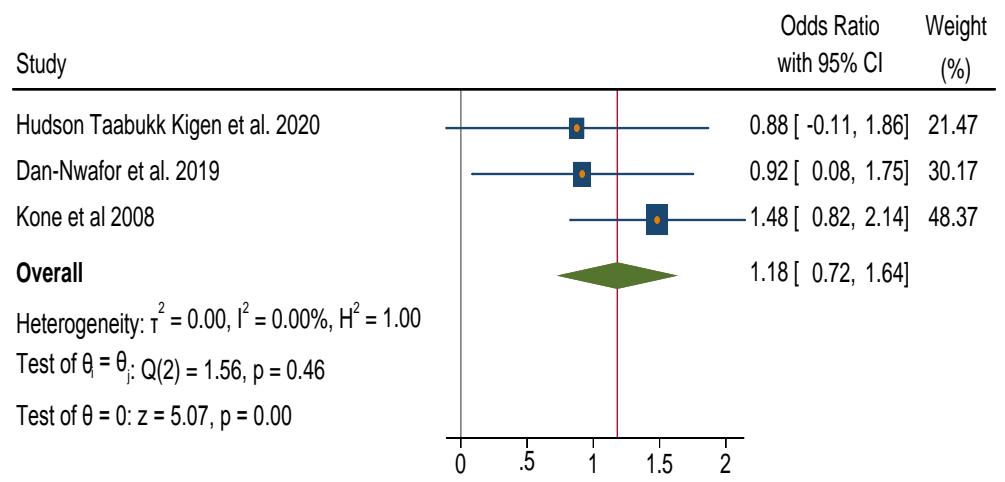

Random-effects REML model

Figure 7. A Forest plot of odds ratio estimates of a cholera outbreak due to a lack of health education on preventive measures. 


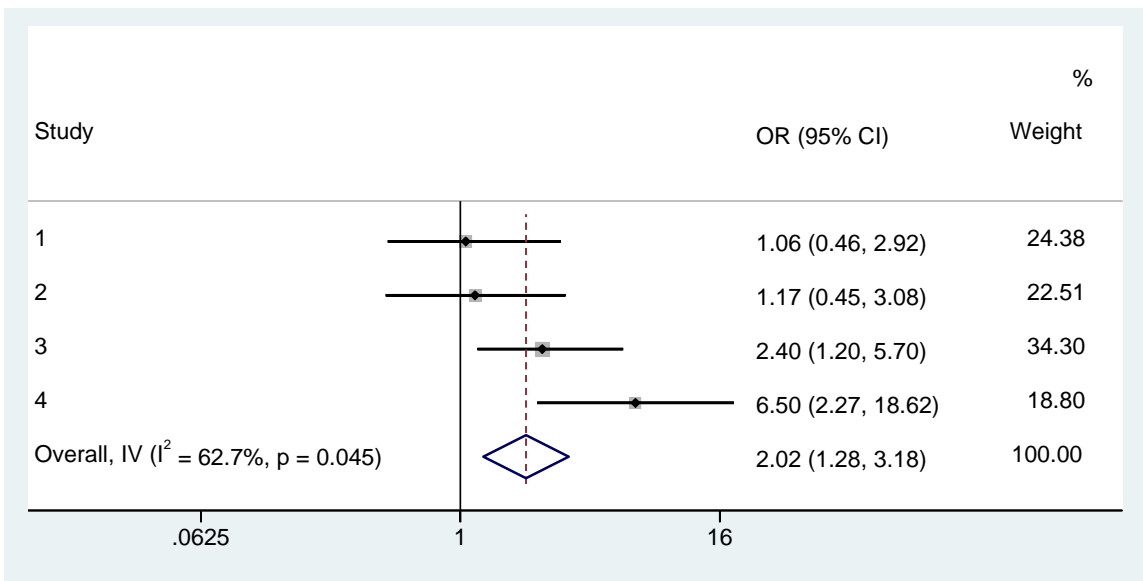

Figure 8. Forest plot of odds ratio estimates of a cholera outbreak due to eating outside of the home.

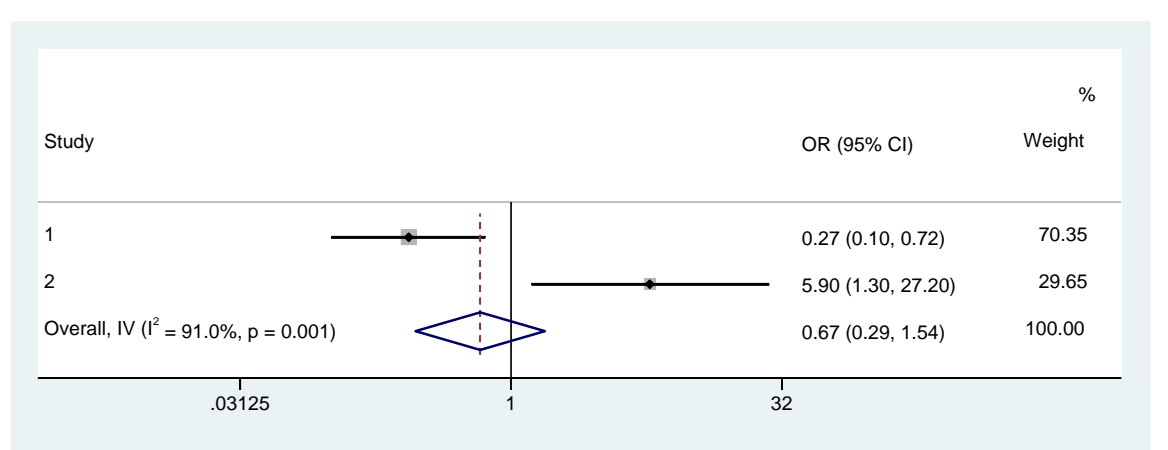

Figure 9. Forest plot of odds ratio estimates of a cholera outbreak due to poor hand hygiene before eating.

\section{Bias in Publication}

The funnel plot and Egger's test were used to screen for publication bias among the included studies for the meta-analysis [41]. According to Egger's test $(\mathrm{P}=$ 0.25 ), the publication bias was shallow, which was not significant to the current data, and funnel plots were symmetrical.

\section{Discussion}

Sporadic cholera outbreaks continue to occur, especially in Sub-Saharan Africa, and are unchecked [42].

Inadequate health literacy, inadequate water and sanitation services, community mobilization, and the absence of national plans, cross-border partnerships, and overcrowding are all risk factors for cholera [43] [44].

In addition to the current preventive steps, improved water, sanitation, and hygiene facilities and the use of OCVs are prominent. In Sub-Saharan Africa, however, there are outbreaks and occasional cases of cholera [45]. To the best of our understanding, no surveys have attempted to quantify the global prevalence of the burden. Therefore, we used a systematic study and meta-analysis to investigate why the incomplete eradication of cholera diseases in Sub-Saharan Africa. 
Our report screened 42 full-text studies after eliminating duplicates and searched for eligibility requirements in the papers we collected from databases applied to the review objectives [46] [47]. Finally, 11 papers from three different research designs were included in the final systematic review and meta-analysis. Many studies [43] [48] used an unmatched case-control design, while three used matched case-control, one retrospective matched case-control, and one case-control design. The sample size for the participants ranged from 47 to 300, and all of the studies included case and control groups. Drinking untreated water, consuming raw vegetables, interacting with infectious people, eating outside of the home, sharing toilet facilities with more than one household, a lack of health education on preventive measures/intervention, and poor hand hygiene before eating were the risk factors found in the studies.

Related research on the risk factors for symptomatic cholera infection identified environmental factors, socioeconomic factors, and intrinsic patient factors [49]. Another study represented the risk and protection hypothesis, and expected protective factors are not as reliably protective as predicted. However, among the variables in safety are cholera transmission through multiple routes and variation in the standard of sanitation and hygiene (WASH) intervention implementation [50]. The correlation between untreated drinking water and cholera outbreaks was identified in seven studies for meta-analyses, and five countries share it. Nigeria, Ethiopia, Cameroon, Kenya, and Zambia are among them. Although the pooled odds ratio estimate is 1.83 , the variability associated with this risk factor for a cholera outbreak was less than $75 \%$. With a long-term solution to water WASH, a report on climate change as a negative or positive related factor for cholera transmission. Drought, for example, can increase pathogen desiccation and reduce rainwater harvesting [51]. Finally, we have four studies that point to consuming raw vegetables as a risk factor for cholera outbreaks. The pooled odds ratio was 1.92, and the study revealed that the risk of the cholera outbreak was nearly twice as high among people who consumed raw vegetables.

A positive correlation was found in a study to evaluate and synthesize established evidence of OCV efficacy when used reactively in real-world conditions [52]. Another risk factor for cholera is fetal, neonatal, and maternal mortality caused by cholera during pregnancy. One research, however, found no difference in fetal death rates by trimester [52]. Contact with an infected individual was listed as a risk factor associated with cholera outbreaks in seven articles in our research. The pooled odds ratio was 1.782, indicating that people are almost twice as likely to acquire the disease if they contact infected people. Four papers from four different countries mentioned sharing toilet facilities with more than one household, and the pooled odds ratio estimate 1.09 as correlated with this risk factor for the cholera outbreak. Three articles identified a lack of health education on preventive measures/interventions as a factor associated with cholera outbreaks among African populations. Just two of the included studies pub- 
lished on poor hand hygiene as a risk factor for cholera, and both were performed in Nigeria.

\section{Minimizing Cholera in Sub-Saharan Africa}

Cholera typically causes significant outbreaks and epidemics in Sub-Saharan Africa, owing to a lack of safe water and sanitation and insufficient case care. When symptomatic or asymptomatic carrier enters a vulnerable community or when an individual meets contaminated environmental sources, an outbreak might begin in a new place [53]. Once cholera has infected individuals in a new region, initial propagation is determined by the degree of individual bacterial shedding, host and organism characteristics, and the probability of other humans encountering an infectious dose. Shedding may occur before and up to seven months after the onset of symptoms. Symptomatic individuals shed significantly more $\mathrm{V}$. cholera $\left(10^{7}-10^{9}\right.$ per $\mathrm{ml}$ of feces) than asymptomatic individuals $\left(10^{3} \mathrm{~V}\right.$. cholera/ml). The infectious dose is $10^{4}-10^{11}$ for a susceptible individual. Following patient excretion, V. cholera develops a hyper-infectious state that lasts at least 5 hours in an aqueous environment [54]. Increased virulence or introduction of similarly virulent strains into an immunologically naive population can raise the danger of outbreaks and may have contributed to ongoing epidemics in Africa [55]. The various biological processes that comprise the aquatic environmental reservoir are essential for the epidemic V. cholerae's long-term survival. Numerous organisms, including algae, crustaceans, floating aquatic plants such as water hyacinth, phytoplankton, and zooplankton such as copepods, are commensal with pathogenic vibrios [56]. Vibrios can enter a dormant state and remain in an aquatic environment in reaction to nutrient restriction; subsequent changes in water temperature, salinity, $\mathrm{pH}$, and nutrients can resuscitate vibrios and result in their growth epidemics. Water is very certainly a significant factor in causing cholera outbreaks in Africa, with lagoons and estuaries contributing to coastal sickness and lakes and rivers contributing to interior sickness [57]. Contamination of human drinking water or food sources can significantly accelerate the spread of cholera. Contamination of water sources continues to be the most often reported risk factor for cholera outbreaks in Africa and throughout the world, accounting for 29 percent of 306 risk factor reports between 1995 and 2005. Transmission is possible through drinking contaminated water from lakes, rivers, estuaries, irrigation canals. In Africa, cholera transmission has been linked to river bathing and residential water consumption [58].

However, when stored in open containers within households or during transportation, clean water may get contaminated. Vendors may pollute drinking water during the extraction of water from wells and the transfer to containers purchased. Shallow, uncovered hand-dug wells, which are the primary source of drinking water for many people in Africa, have been implicated with cholera epidemics across Sub-Saharan Africa on multiple occasions [59]. For instance, in 
the Douala slum region of Cameroon, over 70,000 wells are less than 1.5 meters deep and frequently become contaminated by shallow groundwater, which is polluted by sewage and latrine discharge drains. Food can become infected with Vibrio cholera in the natural environment while being prepared or stored. Due to the concentration of vibrios in the gastrointestinal tracts of mollusks, crabs, these kinds of seafood have been consumed linked to cholera outbreaks. Transmission of V. cholera O1 has been linked to the consumption of unwashed raw vegetables and fruits and poor hygiene practices during meal preparation, as observed during fish gutting processes. Food that has been improperly stored and reheated has been proved to carry cholera. In Africa, cooked rice in Guinea, millet gruel in Mali, and pigeon peas in Malawi have been discovered.

Individual or population-level aggravating factors for cholera outbreaks may exist. For example, increased gastric acidity decreases the chance of developing symptomatic cholera and increases the inoculum necessary for sickness. In addition, Hypochlorhydria caused by Helicobacter pylori infections is widespread in many African settings, increasing individual susceptibility to cholera.

Cholera Outbreaks in Africa.

There have been 127 cases of cholera in Africa. Likewise, vitamin A insufficiency, more than protein-calorie malnutrition, may raise the chance of contracting cholera. Global climatic events may affect the previously stated local climatic risk variables at the population level, most notably the El Nio/Southern Oscillation (ENSO) [56]. ENSO can increase the frequency of rain and floods and the temperature, hence exacerbating outbreaks in cholera-prone locations. Political violence can increase the risk of cholera outbreaks by resulting in the collapse of health and sanitation infrastructure, including disruption of food supplies and subsequent malnutrition, destruction of water supplies and road networks, decreased availability of medical personnel, and destruction of health facilities. Liberia's civil war triggered a large cholera outbreak, aggravated by overcrowded refugee camps and the collapse of public health and clinical facilities, a circumstance that also occurred during Sierra Leone's ten-year civil war. Cholera outbreaks related to civil conflicts may spread to neighboring nations, where hundreds of thousands of people may be housed in refugee camps without proper sanitation and hygiene services. Malawi received political refugees following armed strife in Mozambique in 1986, and by October 1990, Mozambican refugees accounted for $10 \%$ of the population; from 1988 to 1990, at least nine cholera outbreaks were documented in these refugee camps. Similar incidents have occurred among internally displaced persons in Kenya during the post-election violence, in Rwanda following a refugee exodus from the Democratic Republic of the Congo, and in a Sudanese refugee camp. Economic problems unrelated to the conflict have played a role in spreading cholera in several African countries. Beginning of the economic crisis in 1998, Zimbabwe has recorded cholera every year [60], with the vast 2008-2009 outbreak being linked partly to the country's catastrophic economic circumstances and the accompanying collapse of public 
health and clinical service systems. Fuel shortages may impede the management of cholera cases, for example, if health personnel cannot visit their clinics or health centers.

In Nigeria, Zambia, Kenya [61], and Guinea-Bissau [61], simple hygiene measures such as handwashing with soap have been proven to protect against cholera. Having access to a clean latrine within or outside the family may also help reduce the risk of contracting cholera. Acidic meals impede the growth of Vibrio cholera. Consuming tomato sauce ( $\mathrm{pH}$ 5.0) was found to protect against symptomatic cholera during an epidemic in Guinea. Similarly, lime juice, which has been utilized as a prophylactic measure in northern Indian regions during the cholera season, had a significant protective effect when added to the sauce, as demonstrated in Guinea-Bissau 1996. Vibrio survival is decreased in foods containing less water and a higher osmolarity, such as dried, salt-preserved, and sugar-preserved foods. It has been demonstrated that consuming dried fish has a protective impact. In Sub-Saharan Africa, risk factors for cholera infection, transmission, and proliferation are highly context-dependent and may vary significantly between sub-regions and geographical areas. In more developed regions, natural contamination via infected seafood or vegetables is more likely to account for cholera cases.

In contrast, in impoverished areas, risk factors such as a lack of potable water and proper sanitation are more likely to account for cases typically caused by humanitarian crises and exacerbated by natural disasters like flooding. In addition, political instability and civil conflict have been demonstrated to increase the risk of cholera outbreaks, particularly among vulnerable populations such as refugees, who have even less access to safe drinking water and sanitation facilities. Finally, climate considerations exacerbate these harsh conditions repeatedly, resulting in more frequent and fatal cholera outbreaks. In Africa, cholera is predominantly a poverty-related disease.

\section{Limitation and Strength}

The analysis has many advantages: To begin, we used a pre-specified protocol for search and data extraction, as well as a quality evaluation of two independent investigators to reduce assessor bias. The pooled estimate was then subjected to sensitivity analysis to assess its robustness. Nevertheless, our systematic review and meta-analysis have some shortcomings: First, subgroup analysis revealed no substantial difference in heterogeneity compared to the overall outcome.

\section{Practices Implication}

Periodic surveillance commitments necessitate disaggregated data, which would be made available weekly or monthly from local settings, to provide analytical data for the cholera control roadmap. Since most cholera outbreaks are periodic, the existence of cholera can provide annual windows of opportunity for regulatory cholera preparedness activities. Surveys are necessary to establish the effec- 
tiveness of intervention in Sub-Saharan Africa and the mode and study selection. There were no variations in terms of other outcomes when comparing the safety and effectiveness of different ranges of ORS for treating dehydration due to cholera. We suggest that mothers have adequate sanitation and water quality knowledge, diarrheal complications such as dehydration be recognized early, and oral rehydration solution (ORS) be used immediately at home. These are useful in the treatment and prevention of cholera and its complications. Mothers' healthy feeding habits for young children will help reduce more than $10 \%$ of childhood diarrheal mortality. Furthermore, good hygienic practices, especially proper hand washing and waste disposal, may help reduce the prevalence of cholera.

\section{Conclusion}

In Sub-Saharan Africa, a novel behavioral change and these techniques are the last line of protection in improving general wellbeing or health by reducing the burden of cholera disease. The cost-effectiveness of the available approaches must be examined. Improved water, sanitation, and hygiene systems and the use of OCVs can help prevent cholera transmission. Analytical data for the cholera control roadmap provides periodic surveillance commitments that necessitate disaggregated data, which would be made available weekly or monthly from local settings. Since cholera outbreaks are usually seasonal, the regulatory community should plan for cholera preparedness measures annually.

\section{Acknowledgements}

Shandong University supported this work.

\section{Conflicts of Interest}

The authors declare no conflicts of interest regarding the publication of this paper.

\section{References}

[1] Committee to Advise on Tropical Medicine and Travel, An Advisory Committee Statement (ACS) (2001) Committee to Advise on Tropical Medicine and Travel (CATMAT). Statement on Hepatitis A Vaccines for Travelers. Canada Communicable Disease Report, 27, 3-12.

[2] M’Bangombe, M., Pezzol, L., Reeder, B., Kabuluzi, S., Msyamboza, K., Masuku, H., et al. (2018) Oral Cholera Vaccine in Cholera Prevention and Control, Malawi. Bulletin of the World Health Organization, 96, 428-435. https://doi.org/10.2471/BLT.17.207175

[3] Ingelbeen, B., Hendrickx, D., Miwanda, B., van der Sande, M., Mossoko, M., Vochten, H., et al. (2019) Recurrent Cholera Outbreaks, Democratic Republic of the Congo, 2008-2017. Emerging Infectious Diseases, 25, 856-864.

https://doi.org/10.3201/eid2505.181141

[4] Sauvageot, D., Saussier, C., Gobeze, A., Chipeta, S., Mhango, I., Kawalazira, G., et al. (2017) Oral Cholera Vaccine Coverage in Hard-to-Reach Fishermen Communities after Two Mass Campaigns, Malawi, 2016. Vaccine, 35, 5194-5200. 
https://doi.org/10.1016/j.vaccine.2017.07.104

[5] Wang, Y., Li, X., Zhou, M., Luo, S., Liang, J., Liddell, C.A., et al. (2016) Under-5 Mortality in 2851 Chinese Counties, 1996-2012: A Subnational Assessment of Achieving MDG 4 Goals in China. Lancet, 387, 273-283. https://doi.org/10.1016/S0140-6736(15)00554-1

[6] Wilson, K.W. and Wakefield, J. (2020) Pointless Spatial Modeling. Biostatistics, 21, e17-e32. https://doi.org/10.1093/biostatistics/kxy041

[7] Burstein, R., Henry, N.J., Collison, M.L., Marczak, L.B., Sligar, A., Watson, S., et al. (2019) Mapping 123 Million Neonatal, Infant and Child Deaths between 2000 and 2017. Nature, 574, 353-358. https://doi.org/10.1038/s41586-019-1545-0

[8] Amicizia, D., Micale, R.T., Pennati, B.M., Zangrillo, F., Iovine, M. and Lecini, E, (2019) Burden of Typhoid Fever and Cholera: Similarities and Differences. Prevention Strategies for European Travelers to Endemic/Epidemic Areas. Journal of Preventive Medicine and Hygiene, 60, E271-E285.

https://doi.org/10.15167/2421-4248/jpmh2019.60.4.1333

[9] Levine, M.M., Nasrin, D., Acácio, S., Bassat, Q., Powell, H., Tennant, S.M., et al. (2020) Diarrhoeal Disease and Subsequent Risk of Death in Infants and Children Residing in Low-Income and Middle-Income Countries: Analysis of the GEMS Case-Control Study and 12-Month GEMS-1A Follow-on Study. Lancet Glob Health, 8, E204-E214. https://doi.org/10.1016/S2214-109X(19)30541-8

[10] Li, Z., Hsiao, Y., Godwin, J., Martin, B.D., Wakefield, J., Clark, S.J., et al. (2019) Changes in the Spatial Distribution of the Under-Five Mortality Rate: Small-Area Analysis of 122 DHS Surveys in 262 Subregions of 35 Countries in Africa. PLoS $O N E, 14$, e0210645. https://doi.org/10.1371/journal.pone.0210645

[11] Amoah, B., Giorgi, E., Heyes, D.J., van Burren, S. and John Diggle, P. (2018) Geostatistical Modelling of the Association between Malaria and Child Growth in Africa. International Journal of Health Geographics, 17, Article No. 7. https://doi.org/10.1186/s12942-018-0127-y

[12] Marchello, C.S., Hong, C.Y. and Crump, J.A. (2019) Global Typhoid Fever Incidence: A Systematic Review and Meta-Analysis. Clinical Infectious Diseases, 68, S105-S116. https://doi.org/10.1093/cid/ciy1094

[13] Annan, K. (2018) Data Can Help to End Malnutrition across Africa. Nature, 555, Article No. 7. https://doi.org/10.1038/d41586-018-02386-3

[14] Elimian, K.O., Musah, A., Mezue, S., Oyebanji, O., Yennan, S., Jinadu, A., et al. (2019) Descriptive Epidemiology of Cholera Outbreak in Nigeria, January-November, 2018: Implications for the Global Roadmap Strategy. BMC Public Health, 19, Article No. 1264. https://doi.org/10.1186/s12889-019-7559-6

[15] Antillón, M., Warren, J.L., Crawford, F.W., Weinberger, D.M., Kürüm, E., Pak, G.D., et al. (2017) The Burden of Typhoid Fever in Low- and Middle-Income Countries: A Meta-Regression Approach. PLoS Neglected Tropical Diseases, 11, e0005376. https://doi.org/10.1371/journal.pntd.0005376

[16] Awofeso, N. and Aldbak, K. (2018) Cholera, Migration, and Global Health-A Critical Review. International Journal of Travel Medicine and Global Health, 6, 92-99. https://dx.doi.org/10.15171/ijtmgh.2018.19

[17] Bwire, G., Mwesawina, M., Baluku, Y., Kanyanda, S.SE. and Orach, C.G. (2016) Cross-Border Cholera Outbreaks in Sub-Saharan Africa, the Mystery behind the Silent Illness: What Needs to Be Done? PLoS ONE, 11, e0156674.

https://doi.org/10.1371/journal.pone.0156674

[18] Azman, A.S., Luquero, F.J., Ciglenecki, I., Grais, R.F., Sack, D.A., et al. (2015) The 
Impact of a One-Dose versus Two-Dose Oral Cholera Vaccine Regimen in Outbreak Settings: A Modeling Study. PLoS Medicine, 12, e1001867.

https://doi.org/10.1371/journal.pmed.1001867

[19] Birmingham, M.E., Lee, L.A., Ndayimirije, N., Nkurikiye, S., Hersh, B.S., Wells, J.G. and Deming, M.S. (1997) Epidemic Cholera in Burundi: Patterns of Transmission in the Great Rift Valley Lake Region. Lancet, 349, 981-985.

https://doi.org/10.1016/S0140-6736(96)08478-4

[20] Black, R.E., Morris, S.S. and Bryce, J. (2003) Where and Why Are 10 Million Children Dying Every Year? Lancet, 361, 2226-2234. https://doi.org/10.1016/S0140-6736(03)13779-8

[21] Prüss, A., Kay, D., Fewtrell, L. and Bartram, J. (2002) Estimating the Burden of Disease from Water, Sanitation, and Hygiene at a Global Level. Environ Health Perspect, 110, 537-542. https://doi.org/10.1289/ehp.110-1240845

[22] Sauvageot, D., Njanpop-Lafourcade, B.-M., Akilimali, L., Anne, J.-C., Bidjada, P., Bompangue, D., et al. (2016) Cholera Incidence and Mortality in Sub-Saharan African Sites during Multi-Country Surveillance. PLoS Neglected Tropical Diseases, 10, e0004679. https://doi.org/10.1371/journal.pntd.0004679

[23] Bekolo, C.E., van Loenhout, J.A., Rodriguez-Llanes, J.M., Rumunu, J., Ramadan, O.P. and Guha-Sapir, D. (2016) A Retrospective Analysis of Oral Cholera Vaccine Use, Disease Severity and Deaths during an Outbreak in South Sudan. Bulletin of the World Health Organization, 94, 667-674. https://doi.org/10.2471/BLT.15.166892

[24] Utazi, C.E., Thorley, J., Alegana, V.A., Ferrari, M.J., Takahashi, S., Metcalf, C.J.E., et al. (2018) High Resolution Age-Structured Mapping of Childhood Vaccination Coverage in Low and Middle Income Countries. Vaccine, 36, 1583-1591. https://doi.org/10.1016/j.vaccine.2018.02.020

[25] Farag, T., Koplan, J.P., Breiman, R.F., Madhi, S.A., Heaton, P.M., Mundel, T., et al. (2017) Precisely Tracking Childhood Death. American Journal of Tropical Medicine and Hygiene, 97, 3-5. https://doi.org/10.4269/ajtmh.16-0302

[26] Dwyer-Lindgren, L., Squires, E.R., Teeple, S., Ikilezi, G., Allen Roberts, D., Colombara, D.V., et al. (2018) Small Area Estimation of Under-5 Mortality in Bangladesh, Cameroon, Chad, Mozambique, Uganda, and Zambia Using Spatially Misaligned Data. Population Health Metrics, 16, Article No. 13.

https://doi.org/10.1186/s12963-018-0171-7

[27] Deen, J., Mengel, M.A. and Clemens, J.D. (2020) Epidemiology of Cholera. Vaccine, 38, A31-A40. https://doi.org/10.1016/j.vaccine.2019.07.078

[28] De Wever, A., Muylaert, K., Van der Gucht, K., Pirlot, S., Cocquyt, C., Descy, J.-P., et al. (2005) Bacterial Community Composition in Lake Tanganyika: Vertical and Horizontal Heterogeneity. Applied and Environmental Microbiology Journal, 71, 5029-5037. https://doi.org/10.1128/AEM.71.9.5029-5037.2005

[29] Conner, J.G., Teschler, J.K., Jones, C.J. and Yildiz, F.H. (2016) Staying Alive: Vibrio cholerae's Cycle of Environmental Survival, Transmission, and Dissemination. Microbiology Spectrum, 4. https://doi.org/10.1128/microbiolspec.VMBF-0015-2015

[30] Bwire, G., Ali, M., Sack, D.A., Nakinsige, A., Naigaga, M., Debes, A.K., et al. (2017) Identifying Cholera "Hotspots" in Uganda: An Analysis of Cholera Surveillance Data from 2011 to 2016. PLoS Neglected Tropical Diseases, 11, e0006118. https://doi.org/10.1371/journal.pntd.0006118

[31] Bwire, G., Munier, A, Ouedraogo, I., Heyerdahl, L., Komakech, H., Kagirita, A., et al. (2017) Epidemiology of Cholera Outbreaks and Socioeconomic Characteristics of 
the Communities in the Fishing Villages of Uganda: 2011-2015. PLoS Neglected Tropical Diseases, 11, e0005407. https://doi.org/10.1371/journal.pntd.0005407

[32] Bwire, G., Sack, D.A., Almeida, M., Li, S., Voeglein, J.B., Debes, A.K., et al. (2018) Molecular Characterization of Vibrio cholerae Responsible for Cholera Epidemics in Uganda by PCR, MLVA and WGS. PLoS Neglected Tropical Diseases, 12, e0006492. https://doi.org/10.1371/journal.pntd.0006492

[33] Burstein, R., Wang, H., Reiner Jr., R.C. and Hay, S.I. (2018) Development and Validation of a New Method for Indirect Estimation of Neonatal, Infant, and Child Mortality Trends Using Summary Birth Histories. PLoS Medicine, 15, e1002687. https://doi.org/10.1371/journal.pmed.1002687

[34] Burke, M., Heft-Neal, S. and Bendavid, E. (2016) Sources of Variation in Under-5 Mortality across Sub-Saharan Africa: A Spatial Analysis. The Lancet Global Health, 4, e936-e945. https://doi.org/10.1016/S2214-109X(16)30212-1

[35] Weil, A.A., Chowdhury, F., Khan, A.I., Leung, D.T., Uddin, T., Begum, Y.A., et al. (2012) Frequency of Reexposure to Vibrio Cholerae O1 Evaluated by Subsequent Vibriocidal Titer Rise after an Episode of Severe Cholera in a Highly Endemic Area in Bangladesh. The American Society of Tropical Medicine and Hygiene, 87, 921-926. https://doi.org/10.4269/ajtmh.2012.12-0323

[36] Wakefield, J., Fuglstad, G.A., Riebler, A., Godwin, J., Wilson, K. and Clark, S.J. (2019) Estimating Under-Five Mortality in Space and Time in a Developing World Context. Statistical Methods in Medical Research, 28, 2614-2634. https://doi.org/10.1177\%2F0962280218767988

[37] Macharia, P.M., Giorgi, E., Thuranira, P.N., Joseph, N.K., Sartorius, B., Snow, R.W. and Okiro, E.A. (2019) Sub National Variation and Inequalities in Under-Five Mortality in Kenya since 1965. BMC Public Health, 19, Article No. 146. https://doi.org/10.1186/s12889-019-6474-1

[38] Li, B., Tester, M. and Gilliham, M. (2017) Chloride on the Move. Trends in Plant Science, 22, 236-248. https://doi.org/10.1016/j.tplants.2016.12.004

[39] Kiragu, K., Collins, L., Von Zinkernagel, D. and Mushavi, A. (2017) Integrating PMTCT Into Maternal, Newborn, and Child Health and Related Services: Experiences From the Global Plan Priority Countries. Journal of Acquired Immune Deficiency Syndromes, 75, S36-S42. https://doi.org/10.1097/QAI.0000000000001323

[40] Khonje, A., Metcalf, C.A., Diggle, E., Mlozowa, D., Jere, C., Akesson, A., et al. (2012) Cholera Outbreak in Districts around Lake Chilwa, Malawi: Lessons Learned. Malawi Medical Journal, 24, 29-33.

[41] Ferreras, E., Matapo, B., Chizema-Kawesha, E., Chewe, O., Mzyece, H., Blake, A., et al. (2019) Delayed Second Dose of Oral Cholera Vaccine Administered before High-Risk Period for Cholera Transmission: Cholera Control Strategy in Lusaka, 2016. PLoS ONE, 14, e0219040. https://doi.org/10.1371/journal.pone.0219040

[42] Horton, S., Gelband, H., Jamison, D., Levin, C., Nugent, R. and Watkins, D. (2017) Ranking 93 Health Interventions for Low- and Middle-Income Countries by Cost-Effectiveness. PLOS ONE, 12, e0182951. https://doi.org/10.1371/journal.pone.0182951

[43] Graetz, N., Friedman, J., Osgood-Zimmerman, A., Burstein, R., Biehl, M.H., Shields, C., et al. (2018) Mapping Local Variation in Educational Attainment across Africa. Nature, 555, 48-53. https://doi.org/10.1038/nature25761

[44] Golding, N., Burstein, R., Longbottom, J., Browne, A.J., Fullman, N., Osgood-Zimmerman, A., et al. (2017) Mapping Under-5 and Neonatal Mortality in Africa, 2000-15: A Baseline Analysis for the Sustainable Development Goals. Lancet, 
390, 2171-2182. https://doi.org/10.1016/S0140-6736(17)31758-0

[45] Nkoko, D.B., Giraudoux, P., Plisnier, P., Tinda, A.M., Piarroux, M., Sudre, B., et al. (2011) Dynamics of Cholera Outbreaks in Great Lakes Region of Africa, 1978-2008. Emerging Infectious Diseases, 17, 2026-2034.

[46] Horton, R. (2018) Offline: In Defence of Precision Public Health. Lancet, 392, 1504. https://doi.org/10.1016/S0140-6736(18)32741-7

[47] Gurusamy, P.S.R. and Janagaraj, P.D. (2018) A Success Story: The Burden of Maternal, Neonatal and Childhood Mortality in Rwanda-Critical Appraisal of Interventions and Recommendations for the Future. African Journal of Reproductive Health, 22, 9-16.

[48] Grandesso, F., Kasambara, W., Page, A.L., Debes, A.K., M’bang’ombe, M., Palomares, A., et al. (2019) Effectiveness of Oral Cholera Vaccine in Preventing Cholera among Fishermen in Lake Chilwa, Malawi: A Case-Control Study. Vaccine, 37, 3668-3676. https://doi.org/10.1016/j.vaccine.2019.05.044

[49] Wolfe, M., Kaur, M., Yates, T., Woodin, M. and Lantagne, D. (2018) A Systematic Review and Meta-Analysis of the Association between Water, Sanitation, and Hygiene Exposures and Cholera in Case-Control Studies. American Journal of Tropical Medicine and Hygiene, 99, 534-545. https://doi.org/10.4269/ajtmh.17-0897

[50] Jones, N., Bouzid, M., Few, R., Hunter, P. and Lake, I. (2020) Water, Sanitation and Hygiene Risk Factors for the Transmission of Cholera in a Changing Climate: Using a Systematic Review to Develop a Causal Process Diagram. Journal of Water and Health, 18 145-158. https://doi.org/10.2166/wh.2020.088

[51] Musekiwa, A. and Volmink, J. (2011) Oral Rehydration Salt Solution for Treating Cholera: $\leq 270 \mathrm{mOsm} / \mathrm{L}$ Solutions vs $\geq 310 \mathrm{mOsm} / \mathrm{L}$ Solutions. Cochrane Database of Systematic Reviews, No. 12, Article No. Cd003754. https://doi.org/10.1002/14651858.CD003754.pub3

[52] Schwerdtle, P., Onekon, C.-K. and Recoche, K. (2018) A Quantitative Systematic Review and Meta-Analysis of the Effectiveness of Oral Cholera Vaccine as a Reactive Measure in Cholera Outbreaks. Prehospital and Disaster Medicine, 33, 2-6. https://doi.org/10.1017/S1049023X17007166

[53] Nelson, E.J., Harris, J.B., Morris Jr., J.G., Calderwood, S.B. and Camilli, A. (2009) Cholera Transmission: The Host, Pathogen and Bacteriophage Dynamic. Nature Reviews Microbiology, 7, 693-702. https://doi.org/10.1038/nrmicro2204

[54] Glenn Morris, J. (2011) Cholera-Modern Pandemic Disease of Ancient Lineage. Emerging Infectious Diseases, 17, 2099-2104.

https://doi.org/10.3201/eid1711.111109

[55] Piarroux, R. and Faucher, B. (2012) Cholera Epidemics in 2010: Respective Roles of Environment, Strain Changes, and Human-Driven Dissemination. Clinical Microbiology and Infection, 18, 231-238. https://doi.org/10.1111/j.1469-0691.2012.03763.x

[56] Reyburn, R., Kim, D.R., Emch, M., Khatib, A., von Seidlein, L. and Ali, M. (2011) Climate Variability and the Outbreaks of Cholera in Zanzibar, East Africa: A Time Series Analysis. American Journal of Tropical Medicine and Hygiene, 84, 862-869. https://doi.org/10.4269/ajtmh.2011.10-0277

[57] Rebaudet, S., Sudre, B., Faucher, B. and Piarroux, R. (2013) Cholera in Coastal Africa: A Systematic Review of Its Heterogeneous Environmental Determinants. The Journal of Infectious Diseases, 208, S98-S106. https://doi.org/10.1093/infdis/jit202 
[58] Acosta, C.J., Galindo, C.M., Kimario, J., Senkoro, K., Urassa, H., Casals, C., et al. (2001) Cholera Outbreak in Southern Tanzania: Risk Factors and Patterns of Transmission. Emerging Infectious Diseases, 7, 583-587.

https://doi.org/10.3201/eid0707.017741

[59] Adagbada, A.O., Adesida, S.A., Nwaokorie, F.O., Niemogha, M.T. and Coker, A.O. (2012) Cholera Epidemiology in Nigeria: An Overview. Pan African Medical Journal, 12, Article No. 59.

[60] Mukandavire, Z., Liao, S., Wang, J., Gaff, H., Smith, D.L. and Morris Jr., J.G. (2011) Estimating the Reproductive Numbers for the 2008-2009 Cholera Outbreaks in Zimbabwe. Proceedings of the National Academy of Sciences of the United States of America, 108, 8767-8772. https://doi.org/10.1073/pnas.1019712108

[61] Mahamud, A.S., Ahmed, J.A., Nyoka, R., Auko, E., Kahi, V., Ndirangu, J., et al. (2011) Epidemic Cholera in Kakuma Refugee Camp, Kenya, 2009: the Importance of Sanitation and Soap. The Journal of Infection in Developing Countries, 6, 234-241.

https://doi.org/10.3855/jidc. 1966 